\title{
Bernhard Riemann, the Ear, and an Atom of Consciousness
}

\author{
Andrew Bell ${ }^{1} \cdot$ Bryn Davies $^{2}$ (D) Habib Ammari ${ }^{2}$
}

Accepted: 9 July 2021 / Published online: 29 July 2021

(C) The Author(s) 2021

\begin{abstract}
Why did Bernhard Riemann (1826-1866), arguably the most original mathematician of his generation, spend the last year of life investigating the mechanism of hearing? Fighting tuberculosis and the hostility of eminent scientists such as Hermann Helmholtz, he appeared to forsake mathematics to prosecute a case close to his heart. Only sketchy pages from his last paper remain, but here we assemble some significant clues and triangulate from them to build a broad picture of what he might have been driving at. Our interpretation is that Riemann was a committed idealist and from this philosophical standpoint saw that the scientific enterprise was lame without the "poetry of hypothesis". He believed that human thought was fundamentally the dynamics of "mind-masses" and that the human mind interpenetrated, and became part of, the microscopic physical domain of the cochlea. Therefore, a full description of hearing must necessarily include the perceptual dimensions of what he saw as a single manifold. The manifold contains all the psychophysical aspects of hearing, including the logarithmic transformations that arise from Fechner's law, faithfully preserving all the subtle perceptual qualities of sound. For Riemann, hearing was a unitary physical and mental event, and parallels with modern ideas about consciousness and quantum biology are made. A unifying quantum mechanical model for an atom of consciousness-drawing on Riemann's mind-masses and the similar "psychons" proposed by Eccles-is put forward.
\end{abstract}

Keywords Bernhard Riemann $\cdot$ Hearing $\cdot$ Manifold $\cdot$ Mind-mass $\cdot$ Psychon Consciousness · Quantum mechanics

\section{Introduction}

Bernhard Riemann was an outstandingly original thinker whose insights have profoundly shaped mathematics and science, most notably in laying the foundations of modern complex analysis, contributing to the inception of analytic number theory, and making fundamental contributions to the study of higher-dimensional differential geometry. As a result,

Bryn Davies

bryn.davies@sam.math.ethz.ch

1 College of Health \& Medicine, Australian National University, Canberra, Australia

2 Department of Mathematics, ETH Zurich: Eidgenossische Technische Hochschule Zurich, Zurich, Switzerland 
his inspired work continues to be a rich source of mathematical and scientific investigation (Pesic, 2014). Our intent is not to address all these aspects here. Instead, we wish to try and understand why this penetrating thinker spent the last year of his life investigating human hearing. His last paper, only partially complete, attempted to lay out a fresh approach to how that intriguing organ functions (Riemann, 1984). It gives a tantalising glimpse into a new paradigm, one competing with the orthodox model put forward by the eminent Helmholtz, whose reputation then dominated the field. Riemann was here an outsider, and among the ensuing strong intellectual debate, Helmholtz was prompted to privately use the words 'amateur' and 'crank' when referring to his contemporary (Gallagher, 1984; Pesic, 2013).

Riemann's last fragmentary work was only translated into English in 1984 (Riemann, 1984). It is far from a complete and coherent account. Nevertheless, it is the key document in trying to work out what this creative individual was thinking, and why it deserved his last energies. In different ways, the present authors are also involved in understanding aspects of how the ear works, and all three of us are convinced that Riemann was pointing to something important. Riemann was deeply intuitive, conceived multiple novel mathematical ideas, and strongly engaged with philosophical and theological questions beyond mathematics and physics (Gallagher, 1984; Papadopoulos, 2017; Plotnitsky, 2017; Riemann, 1900).

Here, we try to bring together a number of diverse strands of Riemann's thinking into a consistent narrative, hopefully creating a framework from which further investigation might proceed. There are a number of aspects that Riemann got absolutely right, as recent studies of the human cochlea have shown, and these encourage closer inspection. Based on his geometrical investigations, he also made speculations about how one might establish the foundations of hearing, a striking idea in the light of modern research. The following text explores some of the main themes that emerge from Riemann's work on philosophy, mathematics, and physics, themes which shine a light on what may have been behind his last intellectual endeavour. As noted by others, Riemann's last work has unfortunately been neglected (Gallagher, 1984). We will need to triangulate from various hints, but the picture that emerges is that, far from being a crank, Riemann may have been a most prescient thinker.

\section{The Mechanism of the Ear}

After reading Riemann's last fragmentary contribution, Mechanik des Ohres (Riemann, 1984), the modern reader may be left feeling puzzled. We recognise a strong conviction that existing hearing theory is in some way deeply wrong, but the core of the problem, and its proposed solution, is far from clear. Riemann begins by taking the big picture, looking at the scientific enterprise itself and tackling the whole issue of how new knowledge is acquired. In philosophical terms, his concern is that of epistemology. He is clearly concerned that science at the time-based on Newtonian notions of causality-was heading in the wrong direction. He draws a distinction between Newton's approach, which he characterizes as 'synthetic', and that of the philosopher Herbart, which he calls 'analytic'. Riemann had in fact studied Herbart's work closely (Ehm, 2010; Scholz, 1982), and one can say they both subscribed to a similar idealistic outlook, with roots reaching back to Plato (Gallagher, 1984). One can therefore begin to see where Riemann's passion and conviction 
is coming from, and in a sense his motivation is an almost religious necessity to proclaim truth over falsehood.

In other words, we regard Riemann's Mechanism paper as part of a philosophical debate between idealism and materialism. Simply put, Riemann's idealism can be characterised as drawing on the primary concepts of mind and soul, whereas the materialism he is countering ultimately sees the world as the ceaseless jostling of atoms in the void. In modern terms, Riemann wants to view things as top-down, whereas the thinking he wants to oppose see things as bottom-up. At the top of Riemann's hierarchy are mind, soul, and ultimately God, whereas at the bottom are individual atoms. Among modern thinkers who have entertained a not dissimilar notion are neuroscientists like John Eccles, although his dualist interactionism (Eccles, 1994; Popper \& Eccles, 1977) is currently out in favour in philosophical circles (Chalmers, 1996). The details are naturally more complex, and we will pick up on some of the issues below, but this contrast in outlooks is enough to move our project forward. Nevertheless, we choose not to take up the broad distinction between analytic and synthetic which Riemann offers. These labels have been used in philosophy in various ways from one century to the next, and in our view they tend to confuse rather than clarify.

Later discussion will make clearer why Riemann's idealism caused him to object so strongly to Newtonian materialism, but the core of the matter is set out on the first page of Riemann's text: We do not-as Newton proposes-completely reject the use of analogy (the "poetry of hypothesis") (Riemann, 1984: p. 32). ${ }^{1}$ Newton's well-known statement that we must keep to the facts and not deal in hypotheticals clearly antagonised Riemann who saw the human mind as the centre of everything: it is the essential starting point for framing notions of the self and its place in the world. For him, mind (Geistes) and soul (Seele) were basic givens, and its natural mode of operation is to deal with mental constructs, particularly the framing of hypotheses. Science cannot get started-it is lame, as Einstein once said in connection with religion-without taking this entity as its foundation. In this way, Riemann seems to be saying that science needs hypotheses as much as the human soul needs poetry. As we will set out more fully below, the relevance to the ear and its functioning is that hearing must be seen as a multidimensional process by which the mind apprehends a sound. Thus, hearing, according to Riemann, is the result of a mental act, a psychophysical process of perceiving all the subtle tonal qualities of a sound, and that hearing is much more than just the silent vibrations of atoms in the void, as a materialist might view it.

This is where Hermann Helmholtz enters the picture, for he too was concerned with sticking to "the facts" (Pesic, 2014). Helmholtz was a pre-eminent scientist of the day and was highly regarded for his work in physiology, physics, and mathematics. Crucially, however, his philosophy was materialistic (Agutter \& Wheatley, 2008; Hergenhahn, 2009), ${ }^{2}$ and it is perhaps this characteristic that pitted him against the religiously inclined Riemann, who was the son of a pastor and who studied theology before shifting to mathematics. Riemann read much philosophy, including German Naturphilosophie which was a strong current at the time (Ehm, 2010; Scholz, 1982), ${ }^{3}$ moved in a Herbartian philosophical circle

\footnotetext{
1 German: Dichten von Hypothesen.

2 Agutter and Wheatley say that Helmholtz put his name to "virtually a mechanistic materialist manifesto" (p. 124), a manifesto spelled out in Hergenhahn at p. 237.

3 A concise statement of Naturphilosophie comes from Bernfeld (1949): "Naturphilosophie is the name of the pantheistic monism, close to mysticism, professed by Shelling. The Universe, Nature, is one vast
} 
(Gallagher, 1984), and wrote 'Philosophical Fragments' (Riemann, 1900), which we will draw upon later. The particular issue which set them against each other, however, was contained in Helmholtz's monumental work on the physiology of hearing in which he framed his resonance theory of hearing (Helmholtz, 1875). ${ }^{4}$ In part of this work, Helmholtz attempted to show that the three middle ear bones were linked by a kind of ratchet mechanism, an arrangement which meant there was a degree of hysteresis-introducing nonlinearities and distortions - in the way the bones transmitted motion (sound) from the eardrum to the cochlea. On the basis of his anatomical dissection and measurements, Helmholtz claimed that this ratchet was the reason why, when two tones are played, we hear not only those tones but also a third tone-a Tartini tone or intermodulation product—as well. ${ }^{5}$

The idea of a discontinuous ratchet in the middle ear aggravated Riemann. "I am frequently compelled to oppose the conclusions that Helmholtz draws from his experiments and observations", he said, before acknowledging "the great merits of his work." Interestingly, then, the dispute was over the function of the middle ear, not the cochlea. Over the centuries, the spiral-shaped organ has been the focus of many competing theories, which evoke contention even to this day (Dallos, 2003; Raufer et al., 2019; Wever, 1949), but the role the middle ear plays has usually been regarded as secondary, a matter of simple coupling. Why should mere middle ear function engage the interest of a great mathematician?

\section{From the Middle Ear to the Mind}

While Riemann's death, at age 39, cut short the development of his ideas about the mathematical foundations of hearing, his idealist stance prompted him to set out some significant biophysical ideas during his final working days. He was at pains to make clear in his last paper that there must be a one-to-one mapping between the external sound wave and the signal which is transduced in the cochlea and subsequently perceived by the conscious mind. If there was an intervening ratchet mechanism, as Helmholtz proposed, then this mapping would be severely compromised. For Riemann, the existence of a mind and its perception of underlying mathematical patterns and structure must underlie the scientific enterprise. The human mind is continuous with the physical universe, so the power of the mind to create thoughts and hypotheses comes before any causal power attributed to vibrating molecules in the air. For the scientist, the "problem of the organ", as he put it when referring to the function of the cochlea, is to maintain continuity and provide a faithful interface between the sound wave and the apprehending mind, and the same logic applies to the submicroscopic motions of the middle ear.

Footnote 3 (continued)

organism... mind being only the reflection of this unconscious turmoil" (p. 173 of "Freud's scientific beginnings", American Imago 6(3): 163-196.

$41^{\text {st }}$ German edition 1863; 1st English edition 1870 . Helmholtz's work is specifically referred to in Riemann (1984) which is a translation of writings from before Riemann's death in 1866 but before Helmholtz's work appeared in English.

5 Following Riemann's death and the publication of Mechanik des Ohres in 1866, Helmholtz held to the ratchet mechanism, describing it in detail in Pflüger's Archiv of 1869 (translated by J. Hinton as The mechanism of the ossicles and the membrana tympani, New Sydenham Society series 62: 97-155 (1874). Helmholtz says "examination of the mechanism of the joint and the fastening of the ossicles [indicates a solution that] is certainly very different from what the celebrated mathematician appears to have believed", at p. 102. 
Riemann applied this view to ideas far beyond the structure of hearing and went so far as to contemplate, as Pesic describes, "using his many-dimensional curved manifolds as the framework for a grand unified theory of all physical forces" (Pesic, 2014). Pesic goes on to say that Riemann probably intended to use "some kind of multidimensional manifold" to represent hearing (loc. cit.).

His ambitious proposal has aged well during the century and a half of scientific investigation that has followed. Not only are his ideas on the underlying structure of hearing broadly consistent with modern advances-as we shall explore in the next section-but several other fields have similarly converged on manifold-based formulations. Most famously, Einstein's theories of relativity combine space and time into a four-dimensional 'spacetime' manifold, yielding an elegant and powerful framework. In the setting of hearing, the details are much less well developed. However, certain characteristics are clear from our current understanding of the auditory system. Additionally, for Riemann, the ideal of a fundamental manifold - which includes time and space and psychophysical attributes as just some of its emergent features-leads to a method of projective invariance from which the features of hearing (such as the necessary linearity of the middle ear) can be inferred.

Riemann's focus on a broad framework that includes the mind is most evident in his 'Philosophical Fragments' (Riemann, 1900, 1995), where he treats the mind as a conglomeration of thoughts — of individual 'mind-masses' (Geistesmassen)—which have intrinsic causal power.

With every simple act of thinking, something permanent, substantial, enters our soul. This substantial somewhat appears to us as a unit but (in so far as it is the expression of something extended in space and time) it seems to contain an inner manifoldness; I therefore name it "mind-mass." All thinking is, accordingly, formation of new mind-masses. (Riemann, 1900: p.198)

Later, he turns to concepts of how thinking and organic life in general are related, and we will discuss this aspect later. Riemann continues in a philosophical vein and points to the work of Herbart and, most notably, Fechner. Another idealist who was part of Riemann's philosophical circle, Gustav Fechner was deeply concerned with trying to understand how all physical stimuli-whether vibration amplitude, sound, light, smell, pressure, or anything else-were transformed into percepts in the mind. One of the real philosophical problems with idealism is how the mind interacts with matter. If they are two separate planes of existence, how is it possible for the noncorporeal mind to act on hard matter and, in reverse, how can solid matter affect the nonmaterial mind? It is a deep philosophical mystery - the longstanding mind-body problem - as to how microscopic vibrations of molecules in the cochlea can be translated into the percept of loudness in the psyche. Fechner's explorations, based on the work of his teacher Ernst Weber, who was also part of the circle, led him to the founding of "psychophysics", which he defined as "the exact science of the functional relations of dependence among body and soul, more generally, between the corporeal and the mental, the physical and the psychological, world" (Fechner). ${ }^{6}$ The term psychophysics is now scientifically respectable, but in the 19th and 20th centuries many scientists considered unwelcome the notion of an independent conscious mind or

\footnotetext{
${ }^{6}$ As quoted in Heidelberger (2004) at p. 192. This work also has useful discussion of the mind-body problem (chapter 5), Fechner's view of mind-body identity (chapter 2.2), and Herbart's psychology (chapter 1.4).
} 
psyche, and that included Helmholtz, who, although interested in psychophysics empirically, had a strong distaste for metaphysics. Certainly, consciousness as an entity was only accepted into respectable scientific discourse in the latter half of the 20th century, about the time that functionalism, as formulated by B. F. Skinner, began to recede (Baars, 2003).

At this point it is also of interest to recognise a similarity between Riemann's "mindmasses" and the "psychons" proposed by Eccles (1994) more than a century later, a parallel which we examine more closely later in the context of quantum mechanics.

Many textbooks have been written on psychophysics, but the fundamental concept on which they are all built is Fechner's law. Drawing on the work of Fechner's teacher, Weber, the Weber-Fechner law states that the conscious mind senses all physical stimuli in a logarithmic way (Heidelberger, 2004, Ch. 6). Riemann appreciated that this logarithmic transformation was an intrinsic part of hearing, and is the reason why loudness, for example, is measured on a logarithmic scale (whereby a tenfold increase in the power of a sound-10 decibels-results in just a doubling of loudness).

At this point it is illuminating to again return to "Philosophical Fragments" (Riemann, 1900) where Riemann takes up notions of epistemology - the theory of how knowledge is acquired-and of causal chains. He begins by defining 'natural science' as the attempt to comprehend nature in terms of precise concepts, an approach he took in his 1854 habilitation lecture "On the hypotheses that lie at the foundations of geometry" (Riemann, 1990), where the properties of physical space are studied mathematically. In the lecture, Riemann begins by arguing that the development of an axiomatic formulation of geometry, which was the focus of present research activities, was leaving "in the dark" many of the important properties, connections, and structures. By way of contrast, Riemann's lecture suggests that by taking a manifold-based view, we can access a powerful, general and intuitive geometrical tool. In Philosophical Fragments, Riemann says we can apply this tool to sensation, making the suggestion, following Herbart and Fechner, that our sensations ("sensethings in definite space and time relations") have qualities of "color, sound, tone, smell, taste, heat or cold ... not existing outside of us" (Riemann, 1900: p. 213, emphasis added). He therefore infers that sensations need to be considered in terms of quantitative relations or measurements (that is, making use of Fechner's law) and that we can learn about perception by studying the manifold structure of the higher-dimensional spaces described by these quantities. He raises questions about the relations between "thought-processes" (Denkprocesse) and physical processes and, intriguingly, lists four physical processes, at the top of the list being "The absorption of elastic fluids by liquids" (Riemann, 1900), ${ }^{7}$ and the last of which is "Galvanic currents," which no doubt means nerve firings.

Adopting this line of thought, a simple extrapolation might be to say that hearing can be described in terms of a single manifold which includes space, time, and, importantly, the conscious mind. Put simply, hearing involves the mind reaching out through the ear to perceive vibrations in the external world. Admittedly, Riemann says this in a roundabout way, but we think it reasonable, based on his other statements, to make this interpretation. In justification, the following text assembles more pointers from Riemann's own writings and links it to some modern research which appears to be taking a similar direction.

${ }^{7}$ p. 202. German: Absorption von elastischen durch liquid Flüssigkeiten. 


\section{Mathematics and the Manifold}

Why Riemann turned his attention to hearing is more understandable if one recognises parallels between his approach and modern investigations into auditory processing - the field of applied mathematics which attempts to build models of the auditory system's remarkable abilities. One feature in common between Riemann and modern signal processing work is that of basic methodology. When trying to understand cochlear function, attempts to replicate human auditory performance almost always treat hearing as a "black box" (Benedetto \& Teolis, 1993; Yang et al., 1992). This scientific methodology is conceptually similar to Riemann's top-down approach (Ritchey, 1991): the behavior of individual components or atoms is secondary to a system's holistic properties and functions. This black-box concept is perhaps most evident in the machine learning field, where the inner workings of the final sound-to-output mapping are, instead of being carefully designed, developed iteratively and made deliberately opaque and unknowable to the human subject.

Unfortunately, since Riemann gave few mathematical details of his ideas on hearing, we are forced to make inferences based on his other writings, particularly in the field of geometry. A key question in acoustics is identifying when two sounds are perceptually similar. Mathematically speaking, this is a question of finding a way to measure the (nonEuclidean) distance between two points in the abstract space of all audible sounds. A typical approach is to project them onto a selected set of basis functions (common examples being windowed Fourier modes or wavelets). In making this projection it is assumed that, at least locally, the space of audible sounds can be fully characterised by the corresponding $n$-vector, meaning that the space of audible sounds is an $n$-manifold. Thus, we need to measure the distance between two points on a higher-dimensional manifold. Given that there is no reason for this space to be Euclidean, we likely need the tools of Riemannian geometry to be able to handle this problem.

Although Riemann never explicitly formulated a manifold-based theory of hearing, he did set out a similar framework for other domains, notably in the theory of colour perception. By the time that Riemann gave his famous 1854 lecture (Riemann, 1990), Helmholtz and Young had developed substantial theories of vision, such as the trichromatic theory of colour perception and the identification of three perceptually independent parameters, now known as hue, saturation, and brightness (Pesic, 2014). Helmholtz had also contemplated how to describe the perceived distance between different colours, speculating that two colours that are an equal distance apart in his sketched diagram of color perception "correspond to equal differences between the logarithms of the wavelengths" (Helmholtz, 1911: p. 54/64). No doubt with this work in mind, Riemann devotes a brief part of his lecture to highlighting that such parameterisations demonstrate that colour is a "multiply extended manifold" (Riemann, 1990). Riemann's approach naturally "raises the question of whether or not the manifold of colour perception is Euclidean in its geometry" (Pesic, 2013, loc. cit.). If a similar generalised formulation of hearing is considered, then the same question arises. While the answer isn't yet clear, some important properties have already been established. For instance, any approach needs to be capable of representing signals in a nonlinear way, to account for the nonlinear mechanisms that are used in the human auditory system. This also allows much greater levels of sophistication and, for example, allows representations to be created that are invariant to certain transformations. If a signal is distorted only slightly, or in a perceptually minor way, then the representation of that signal should 
not change significantly. ${ }^{8}$ However, constructing appropriate representations is not a trivial task and cannot be done using linear representations. ${ }^{9}$ As a result, there has been much interest in nonlinear representations of signals, such as those arising from the scattering transform (Andén \& Mallat, 2014) and other convolutional neural networks. This is an area in which two of us (HA and BD) are currently engaged, an enterprise where we are making efforts to provide some degree of domain reduction of sound signals based on perceptual qualities. ${ }^{10}$ We hope this may ultimately provide a definite example of where Riemann's ideas were heading. It would be over-reach to suggest that Riemann in any way anticipated the development of such advanced techniques; it is noteworthy, however, that not only do these approaches match his geometrical ideas, but also that Riemann was keenly aware of the importance of auditory invariants. Indeed, by considering 'Klang' (or timbre) as such an invariant he was able to forcefully engage with Helmholtz on biomechanical properties of the middle ear-in particular its linearity down to microscopic dimensions.

Riemann wants to insist on a certain phase fidelity, how "the ratio between the synchronous pressure fluctuations of the air and of the inner ear fluid remains virtually constant during a sound" (Riemann, 1984, p. 35). He continues, "We demand... that the transmission mechanism itself produce no gross distortion of timbre," adding that "we believe... its fidelity is much greater than is usually supposed" (loc. cit.). ${ }^{11}$ This quote is a nice way of expressing that there is a one-to-one correspondence between the sound signal in the air and inside the cochlea, and can also be taken as an argument against any theory of cochlear function which introduces appreciable time delay. Moving forward to the 1900s, one wonders what he might have thought of the traveling wave theory of cochlear function which superseded Helmholtz's long-established resonance theory. Championed by Békésy, 1960 (see also Olson et al., 2012), a prime attribute of the traveling wave was that it could explain the apparently long delays-many cycles-observed in cochlear experiments, delays which Helmholtz's resonance theory was at a loss to explain. By itself, a simple resonator can contribute a phase shift of only $\pm 90^{\circ}$, a delay of just a fraction of a cycle (Bell, 2012). Looked at another way, however, the virtue of a simple resonance model of the cochlea is that it can largely preserve the character of the original sound, which is precisely what Riemann was seeking. ${ }^{12}$

Riemann's focus on purity and finesse, to uphold and preserve the manifold, is why we believe Riemann spends considerable time trying to convince the reader that the vibrations in the ear must be submicroscopic. He describes how a foghorn can be heard at a great distance-_four or five English miles"-from which he calculates, via the inverse square law, that the amplitude of vibration at the ear must be "one ten-millionth" of that at the source, motions "so small that they cannot be observed with a microscope" (Riemann, 1984: p. 35). At this scale, the properties of the manifold, including its geometry (and whether it is a differentiable continuum or not) will control the acoustics and possible dynamics. Ultimately, the physics of the ear will depend on whether we consider its internal acoustic

\footnotetext{
${ }^{8}$ For a discussion of invariant representations in the setting of the scattering transform, see Mallat (2012).

9 For example, the only linear, bounded functional that is invariant to translation is the global average operator, which gives little useful information, see e.g. Bruna (2012).

10 Some of our ideas are outlined in Ammari and Davies (2020a).

11 The term "timbre" is used as the translation of the German word "Klang" (footnote 1 of Riemann, 1984), but it can be argued that this term fails to satisfactorily convey what Riemann was driving at; perhaps the word finesse may be better in this context.

12 A reconciliation between the traveling wave and resonance pictures can be achieved by taking account of the difference between phase delay and group delay (see Bell 2012).
} 
medium to be uniform and differentiable or whether it contains discrete shock fronts. Riemann had already demonstrated, by mathematical analysis, that acoustic shock waves must exist, even though it took many decades and much controversy before such waves were experimentally observed (Parpart, 1979). His concern was that loss of fidelity or finesse would destroy the emotion-laden qualities that we perceive in sound.

Recent experimental work has indeed confirmed the minuteness of cochlear transduction processes (Olson et al., 2012), and it has been calculated that at auditory threshold the tips of the hair cell stereocilia must be deflected by only $0.3 \mathrm{~nm}$, representing an angle of just 0.01 degree (Dallos, 1996). The corresponding displacement of the eardrum is then truly microscopic (some... $10^{-10} \mathrm{~m}$ ), about the diameter of the hydrogen atom. There is a species of frog that can detect ground vibrations of about the same magnitude (Bialek, 2012: p. 234). Considerations such as these point towards possible quantum mechanical involvement, and this is an issue we take up later.

\section{Physical Processes in the Ear}

Summarising to this point, Riemann's view is that hearing should be viewed as top-down, not bottom-up, an arrangement in which top is the mind and bottom is matter. On this view, the mind is part of a manifold which reaches out through the ear and perceives vibrations, and the manifold includes all the psychophysical properties that the Weber-Fechner law prescribes. Riemann thought something is missing if we take the view, as Helmholtz did, that vibrations in the ear create a causal cascade of mechanical motions, neural transduction, nerve propagation, and electrical activity in the brain.

As Plotnitsky reminds us in his perspective on Riemann's work (Plotnitsky, 2017), the properties of time and space derived from the manifold relate to the possible behavior of physical objects at the smallest scale. If there is no continuity at the scale of the immeasurably small (Unmessbarkleine), causality may be lost. One possible implication is that the mind can no longer reach out and properly perceive the finest of vibrations in the external world. Here Riemann may want us to feel, in a poetical way, the beauty and finesse of the hearing faculty — of hearing music, the human voice, birdsong — a feeling that disappears when the listener can no longer listen.

When introducing Riemann's unfinished ear paper, his publisher and friend Jacob Henle wrote an introductory paragraph in which he concluded, no doubt from personal conversation, that "Riemann thought that the mathematical problem to be solved was in fact a hydraulic one" (Riemann, 1984: p. 31), and in the light of Riemann's work on shock waves and the fact that the inner ear is filled with fluid, we can begin to appreciate possible implications. Recent work by the present authors has conjectured about the existence of compressible elements within the fluid-filled cochlea (Ammari \& Davies, 2019, 2020b; Bell, 2003, 2005, 2008), a possibility we continue to pursue.

At this juncture it is instructive to return to Philosophical Fragments and look closer at those four physical processes that Riemann believed underlaid all "thought processes". These were the bridge between the outer world and the inner, between the physical and the mental. At the top of the list, as mentioned before, was the "absorption of elastic fluids by liquids", so one might wonder whether this first hydraulic interaction might involve some interplay between compressible and incompressible materials. This possibility is strengthened by his next assertion that "The substance of organisms ... is partly solid (only partly brittle), partly gelatinous, partly liquid or elastic fluids but always porous, i.e., notably 
penetrable by elastic fluids." ${ }^{3}$ This seems an apt description of the composition of the cochlea in which the solid temporal bone is filled with incompressible aqueous fluid and an astounding complexity of sensory tissues. The hairs of the sensing cells touch a gelatinous tectorial membrane, and there is reason to think that the supersensitive outer hair cells themselves may be compressible. All of this reinforces the possibility that, as Riemann thought, sound transduction may involve compression or give rise to shock fronts.

In the context of hydraulic interactions, it is worth noting that a specific model constructed by one of us $(\mathrm{AB})$ conjectures that the micromechanics of the cochlea might perhaps involve specific fluid-structure waves known as Krauklis or "squirting" waves (Bell $\&$ Fletcher, 2004). Krauklis waves are very low velocity ripples which may allow resonant interactions to occur between rows of outer hair cells, in this way allowing the whole organ to operate as a surface acoustic wave (SAW) resonator. Such devices are electronic modules commonly used in signal processing, and have the special property of converting fast electromagnetic waves into slow shear waves on the surface of a substrate. The reduction in speed is typically a factor of $10^{6}$ or more, and so a similar device in the cochlea could possibly convert a fast pressure wave in the fluid $\left(1500 \mathrm{~m} \mathrm{~s}^{-1}\right)$ into a fluid-borne wave propagating between the rows at just millimetres per second, again about a million-fold (Bell, 2014). The initial input stimulus to the outer hair cells is conjectured to occur through the compression of material inside the cells-perhaps with compressibility comparable to that of an air bubble-by incoming sound pressure.

Recent work has looked at the air bubble question theoretically, and the results show that an array of air bubbles, graded in size and surrounded by incompressible fluid, are able to replicate the same "tonotopic" organisation of the cochlea-meaning that the array possesses the same distinctive tuning gradient that the organ displays (Ammari \& Davies, 2019, 2020b). The interaction of an air bubble immersed within incompressible fluid would no doubt have been of keen interest to Riemann.

\section{Quantum Mechanical Possibilities}

As noted earlier, Riemann recognised the atomic-scale of sound transduction, and in this section we set out the possibility that quantum mechanical processes may be involved. This step goes beyond what Riemann conceived at the time, but it does build on his idea that the human mind comprises individual mind-masses and also brings in modern thinking that life and consciousness may actually have quantum mechanical underpinnings (Abbott et al., 2008; Al-Khalili \& McFadden, 2014; Rosenblum et al., 2017). In this light, it is worth considering whether it might be possible to view the cochlea as a quantum detector whose task is to extract a signal from a sea of phonons flooding through the organ.

In constructing a case for quantum mechanical involvement, we draw on two special features. First, there is the parallel between Riemann's mind-masses and the quantum mechanical 'psychons' introduced in the 1980s by the neuroscientist John Eccles, who argued that psychons were atoms of consciousness associated with collections of dendrons in the cortex. Second is the observation that the nervous system is built of both afferent (ascending) and efferent (descending) nerve pathways. Afferents convey action potentials from the

\footnotetext{
13 Combination of translations from (Riemann 1900: p. 203) and (Riemann 1995: p. 53). We consider "elastic fluids" (Keyser) to be a better translation than "gaseous" (Cherry) for the original elastische Flüssigkeiten.
} 
periphery to the cortex, while efferents take signals from higher brain structures down to muscles and sense organs. The roles of each sort of pathway are still being explored, but it is clear there needs to be close coordination of information flow in both directions (Cooper \& Guinan, 2011). The issue is complex, but the extensive efferent pathway is at least consistent with Riemann's notion that the mind "reaches out" to the cochlea to sense information flowing in from the outside world.

One problem with that notion is the slow speed at which nerve impulses travel along the efferent pathway, leading to appreciable delays. However, a key aspect here is the relevance of Benjamin Libet's work on the way the mind, in constructing the numinous present, compensates for all the various delays in information coming and going within the nervous system. Eccles discusses the issue in his book with Popper (Popper \& Eccles, 1977), noting at p. 364 how Libet's work points to the mind projecting the present moment back to a common origin about $200 \mathrm{~ms}$ in the past ("antedating"). In effect, the mind anticipates the present, surfing it as a wave. Eccles says that Libet may have discovered "a temporal adjustment attributable to the self-conscious mind" (op. cit. p. 364).

For our present discussion, the importance of the nervous system having both efferent and afferent pathways, and their rich interconnection, is that networks of sustained reverberating loops can be created (Kistler \& De Zeeuw, 2003). The auditory system contains as many, if not more, efferent connections than afferent, and one might wonder why this might be (Slepecky, 1996). Some have conjectured that reverberating loops may be the basis for perceived tinnitus as well as for the sustained brain activity picked by EEG electrodes (Dornhoffer et al., 2006; Lozano-Soldevilla \& VanRullen, 2019). In terms of the cerebral cortex, another neuroscientist, Szentágothai, has noted the "massive reentrant circuitry" in these higher centres, and has wondered at the "crucial significance" this special modular architecture may have (Szentágothai, 1984). Perhaps an essential function of the intertwined reflex arcs between afferents and efferents might be, he suggests, to establish a "circular chain" of reciprocal connections (Szentágothai, 1979).

In this context we point out that such loops would allow strong feedback processes to occur, and that, as described below, feedback is physically equivalent to "cooling" a system (it narrows the bandwidth, in the process reducing thermal noise). Potentially, therefore, noise might be reduced below thermal levels-and perhaps approach quantum limits. In brief, the suggestion is that consciousness-identified with mind-masses or psychonsmight be generated in the cerebral cortex via quantum phenomena, and a brief outline of this idea gives extra currency to Riemann's thinking.

A good starting point is to consider the work of Bialek, a biophysicist who for a number of years made concerted efforts to investigate the ways by which feedback could reduce thermal noise in physical systems to levels where quantum mechanical limits might apply (Bialek \& Schweitzer, 1985; Bialek, 1983a, 1983b, 1983c, 1984, 1987). In the context of the ear, Bialek and Wit (1984) made the case that the sounds emitted by the cochlea as otoacoustic emissions (and recorded with a sensitive microphone in the ear canal) are already at levels which approach quantum limits. Interestingly, some years later Bialek considered these efforts "a failure" and abandoned the attempt (Bialek, 2012: p. 237). Be that as it may, others have continued to seek a quantum mechanical base for biology and consciousness (Abbott et al., 2008; Al-Khalili \& McFadden, 2014; Georgiev, 2018; Rosenblum et al., 2017), and we believe it would be premature to dismiss this far-reaching hypothesis.

Bialek explains how a physical system can be "cooled" by applying a feedback force proportional to its observed velocity (Bialek, 2012: pp. 233ff); this effectively adds extra drag, and thermal noise is therefore reduced. If the initial temperature is $T$, and the effective 

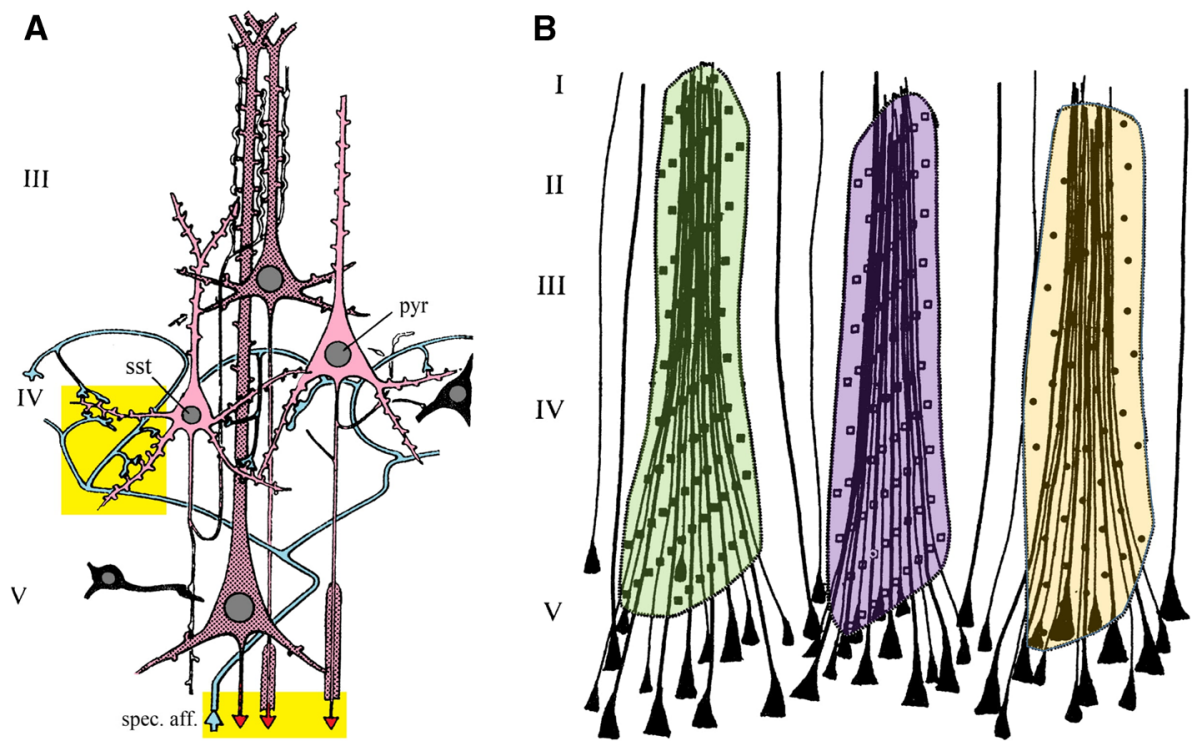

Fig. 1 Proposal for how consciousness may arise, prompted by ideas of Riemann and, more recently, Eccles. a A distinct feature of cerebral cortex (layers III, IV, and V) is that it is the interface between afferent, ascending nerve fibres (in blue) and efferent, descending fibres (red), as highlighted in the bottom yellow square. Afferents touch pyramidal cells (pyr) at synapses highlighted by middle yellow square. The cell thus establishes a feedback loop between afferent and efferent in which amplification will reduce ambient noise, equivalent to reducing the effective temperature (i.e., cooling). It is conjectured that if the feedback gain were sufficiently high, thermal noise might be reduced to the extent that the system becomes quantum noise limited. b Eccles (1990) proposed that these same cortical layers (I-V) were where mind and brain interacted (dualistic interactionism), and he called these quantum-like atoms of the mind psychons (coloured envelopes), entities reminiscent of the mind-masses proposed by Riemann. It is suggested that psychons arise from feedback which effectively cools the system to a level where quantum phenomena can operate. A, from Szentágothai (1979) with permission of MIT Press; B from Eccles (1990) with permission of The Royal Society

drag becomes $\gamma+\eta$ instead of just $\gamma$ (where $\gamma$ is the initial drag and $\eta$ is the feedback parameter), then the effective temperature, $T_{\text {eff }}$, becomes

$$
T_{\text {eff }}=T \gamma /(\gamma+\eta) \text {. }
$$

Thus, by observing the system and applying a feedback force, it is possible to synthesise a system that is effectively colder. For example, Bialek describes how the effective temperature of a suspended mass of $1 \mathrm{~g}$ can, by applying carefully controlled laser feedback, be reduced from room temperature to $7 \times 10^{-3} \mathrm{~K}$. Given this result, it is suggested that an arrangement of reverberating neural loops might, through a feedback mechanism, also be able to reduce the effective temperature of the neurons in the cerebral cortex to a level where thermal effects are reduced and quantum mechanics comes into play. Interestingly, the strategy can work whether the feedback is applied positively or negatively. ${ }^{14}$

\footnotetext{
14 An additional factor to be considered is that nerves are piezoelectric - that is, the diameter of a nerve changes depending on its electrical potential, so that as an action potential passes along a nerve, its diameter momentarily increases (Costa et al., 2018). Piezoelectricity could thus allow electrical feedback to directly stabilise the physical size of a neuron, although of course there may be another special structure in a dendron that is the target for feedback cooling.
} 
The idea can be applied to the cerebral cortex, where anatomy shows pyramidal neurons arranged in five distinct layers (Fig. 1a). A notable feature is the rich interconnectedness between efferent and afferent nerve fibres (yellow squares), providing ample opportunities for active feedback loops to be established. In this way, effective cooling may be possible, and it is suggested that the multiple layers might allow a sequence of cooling stages to take place, just as multi-stage refrigeration is used to achieve temperatures near absolute zero. A feature of the scheme is that consciousness can be readily turned on (we are awake) or switched off (asleep or anesthetised) simply by adjusting the overall system gain, perhaps by a control signal from the thalamus. The proposal is not unlike making a material superconducting by lowering its temperature.

Figure $1 \mathrm{~b}$ illustrates the psychons that Eccles proposed were mental entities arising in assemblies of cortical dendrons (Eccles, 1990). He maintained that psychons manifested from quantum mechanical events, and were a two-way interface between mind and body (Eccles, 1994). While psychons allow sensory stimuli to affect the mind, they also allow the will to exert its effect on the body, creating physical activity. As noted, Eccles' view is currently out of favour (Chalmers, 1996), but our assessment is that his idea is compatible with Riemann's mind-masses and deserves closer investigation. Unlike many philosophical speculations, the model is anatomically well-defined and is open to scientific study. Nevertheless, we acknowledge its limitations. As one reviewer pointed out, it is not clear why psychons should be manifolds in the same way as Riemann's mind-masses, or even why consciousness itself should be a manifold. Finally, it remains speculation that the conscious mind depends on quantum mechanical processes (Adams \& Petruccione, 2021; Georgiev, 2018). We leave these questions for future exploration.

\section{Other Things Riemann Got Right}

It is too early to say whether the above speculations move us closer to the truth of the matter, but they certainly enlarge the field of possible answers. To gain an adequate perspective, it must first be recognised that the hearing field changed dramatically in 1978 when David Kemp published his finding that the human ear not only detects sound but emits it as well (Kemp, 1978). ${ }^{15}$ Otoacoustic emissions have revolutionised our understanding of how the ear works, forcing us to acknowledge that the ear is not a passive, heavily damped microphone but an active, highly tuned signal processing device. There is much research underway into otoacoustic emissions, and our knowledge of cochlear mechanics has improved enormously from using them as a noninvasive window into the inner ear. It is fairly certain that the emissions are generated by the cochlea's outer hair cells, which, drawing on metabolic energy sources and working together, create an active entity now known as "the cochlear amplifier" (Neely, 1983). Using some sort of positive feedback, the cochlear amplifier is able to amplify faint sounds entering the cochlea by $60 \mathrm{~dB}$ or more. Placing a sensitive microphone in the ear canal, it is possible to pick up continuous faint, pure sounds_-spontaneous otoacoustic emissions-which are a by-product of the cochlear

\footnotetext{
15 Interestingly, Kemp's findings were rejected by Nature because the reviewers saw it as 'of limited significance' and 'too specialised a topic' (see Kemp (2008). Kemp's work confirmed Thomas Gold's proposal 30 years earlier that the cochlea might be active and highly tuned, an idea ahead of its time. Kemp's findings inspired one of us $(\mathrm{AB})$ to investigate the properties and origins of these faint, pure sounds.
} 
amplifier at work. Clearly, our understanding of how the whole organ works is still far from complete and much more work is needed (Manley \& Brownell, 2008; Manley et al., 2017).

We cannot say whether Riemann had an inkling that the cochlea was an active signal processing device, or that he would pick up on the quantum mechanical possibilities. Would he view otoacoustic emissions as just the final stage in a subtle interaction between mind and matter? However, it is now clear there are a number of things about the ear which Riemann did get right. In this connection, it helps to keep in mind something that Thomas Gold (1920-2004) learned, from his own hard experience, about the way science progresses: "New ideas in science are not always right just because they are new. Nor are the old ideas always wrong just because they are old" (Gold, 1989). ${ }^{16}$

First then, on the matter of nonlinearity, research has indeed shown that the middle ear is perfectly linear over the scale of normal hearing levels (Hartmann, 1998: p. 511ff). Here, Riemann was correct, and Helmholtz was wrong. At the same time, however, we now know that cochlear transduction is itself nonlinear (Hudspeth, 2014). Thus, when two pure tones of frequency $\mathrm{f} 1$ and $\mathrm{f} 2$ enter the cochlea, a set of distortion products are generated, probably by the outer hair cells, producing frequencies of $\mathrm{mf} 2-\mathrm{nf} 1$, which includes the Tartini tone $2 \mathrm{f} 1-\mathrm{f} 2$. What would Riemann make of the presence of cochlear distortion? What are the implications for the manifold and the mind of the listener? Interestingly, experiments have been done in which the cochlea's distortion products have been electronically cancelled out, and the reported result is that such distortion-free sounds now sound "drab" and lacking musical quality (Goldstein, 1967: p. 684). This outcome suggests that some sort of mapping is going on, and that the process does not appear to rely on perfect fidelity. At the same time, it is now well known that sound transduction involves conversion of sound pressure into action potentials, effectively a transformation from the analog domain to a digital one.

Second, Riemann refers to the third middle ear bone, the stapes, as the "lensbone", a descriptive term uniquely his own. In fact, a large body of research has now shown that the middle ear chain serves to magnify the intensity of sound by about 25 decibels, so that it indeed acts acoustically as a sort of magnifying lens.

Third, Riemann's paper draws attention to the central role of the middle ear muscles in hearing. The muscles, the tensor tympani and the stapedius, attach to the middle ear chain and regulate sound transmission to the cochlea. He draws a parallel to how muscles in the eye control the diameter of the iris and adjust the amount of light falling on the retina, although it is not clear to him how the middle ear muscles control auditory magnification. "I ... have no idea what a continuously variable reflex activity of $M$. tensor tympani is supposed to contribute to the exact comprehension of a piece of music" (Riemann, 1984: p. 35). However, he does make the crucial observations that "the alert ear", for precise hearing, depends upon "the foot of the stirrup [pressing] slightly against the inner ear fluid by tension of $M$. tensor tympani so that the pressure of the inner ear fluid is slightly greater than that of the air in the tympanic cavity", and accordingly that " $M$. tensor tympani establishes the most favorable pressure for accurate hearing" (Riemann, 1984: pp. 37-38).

Here, Riemann seems to be close to the mark. According to the intralabyrinthine pressure (ILP) theory formulated by Gellé and others some decades later (Gellé, 1881),

\footnotetext{
${ }^{16}$ In 1948 Gold introduced the revolutionary idea that the cochlea was active and highly tuned, a concept that was strongly resisted at the time but which Kemp was to prove correct. Gold goes on to say that "uncritical acceptance of the established ideas [can be] a real hindrance to the pursuit of the new. Our period is not going to be all that different in that respect, I regret to say" (p. 103).
} 
hydraulic pressure is the key parameter by which the middle ear muscles control cochlear sensitivity. Riemann does not quite set out that connection explicitly, but he comes close. The ILP theory was current in hearing science at the end of the 19th century, but for various reasons fell out of favour (the current textbook account is that the muscles stiffen the connections between the ossicles of the middle ear and thereby reduce acoustic transmission (Pang \& Peake, 1986)). Recently, the explanatory power of the ILP theory has again been recognised, and a case for reconsidering its merits has been published (Bell, 2011). The ILP theory seems to accord with what Riemann was suggesting, although more research is needed to decide the issue.

Finally, there are the provocative quantum mechanical considerations, and here we cannot be sure of what Riemann would make of them, but the parallel between mind-masses and Eccles' psychons is a matter worth investigating further.

\section{Conclusion}

There is no doubt Riemann was a mathematician of the highest order, but we are also struck that he also seemed to be an idealist of the highest order too, placing thoughts — or "mindmasses"- at the top of the causal chain. Mind-masses are his fundamental atoms taking the place of Cartesian material atoms. He subscribed to the Kantian notion that "What an agent strives to effect must be determined by the idea of the agent" (Riemann, 1984: p. 214) and the causal power of the conscious will is a defining characteristic.

For Riemann, hypotheses were the working machinery by which thoughts engage with the world. All thinking, as he saw it, was the formation of new mind-masses-molecules of thought, we might say - and so hypotheses become the essential stuff at the centre of scientific discourse. As with poetry, the best scientific hypotheses must therefore be sonorous and beautiful, so that, against Newton, we should treasure the 'poetry of hypothesis' as a path to scientific discovery. Riemann says that the word hypothesis now has "a somewhat different significance from that given by Newton" (Riemann, 1984: p. 214), so that by the term we should now understand all thoughts—-mind-masses_connected with phenomena.

At the very beginning of his Philosophical Fragments, Riemann displays an epigraph quoting two lines of Latin by Lucretius, the Roman poet and Epicurean philosopher. Translated into English it makes a fitting conclusion to this survey of Riemann's work on the ear. "Do not scornfully reject the gifts I have devotedly marshalled for you, before you have understood them" (Riemann, 1995: p. 51).

\section{Funding Open Access funding provided by ETH Zurich.}

Open Access This article is licensed under a Creative Commons Attribution 4.0 International License, which permits use, sharing, adaptation, distribution and reproduction in any medium or format, as long as you give appropriate credit to the original author(s) and the source, provide a link to the Creative Commons licence, and indicate if changes were made. The images or other third party material in this article are included in the article's Creative Commons licence, unless indicated otherwise in a credit line to the material. If material is not included in the article's Creative Commons licence and your intended use is not permitted by statutory regulation or exceeds the permitted use, you will need to obtain permission directly from the copyright holder. To view a copy of this licence, visit http://creativecommons.org/licenses/by/4.0/. 


\section{References}

Abbott, D., Davies, P. C. W., \& Pati, A. K. (2008). Quantum aspects of life. Imperial College Press.

Adams, B., \& Petruccione, F. (2021). The light of the mind. Physics World, 34(1), 24-28.

Agutter, P. S., \& Wheatley, D. N. (2008). Thinking about life: The history and philosophy of biology and other sciences. Springer.

Al-Khalili, J., \& McFadden, J. (2014). Life on the edge: The coming of age of quantum biology. Bantam Press.

Ammari H., Davies B. (2020a). A biomimetic basis for auditory processing and the perception of natural sounds. arXiv preprint 2005.12794

Ammari, H., \& Davies, B. (2019). A fully coupled subwavelength resonance approach to filtering auditory signals. Proceedings of the Royal Society of London A, 475, 20190049. https://doi.org/10.1098/rspa. 2019.0049

Ammari, H., \& Davies, B. (2020b). Mimicking the active cochlea with a fluid-coupled array of subwavelength Hopf resonators. Proceedings of the Royal Society A, 476, 20190870. https://doi.org/10.1098/ rspa.2019.0870

Andén, J., \& Mallat, S. (2014). Deep scattering spectrum. IEEE Transactions on Signal Processing, 62(16), 4114-4128.

Baars, B. J. (2003). The double life of B F Skinner: inner conflict, dissociation and the scientific taboo against consciousness. Journal of Consciousness Studies, 10(1), 5-25.

Békésy, G. (1960). Experiments in hearing. McGraw-Hill.

Bell, J. A. (2005). The underwater piano: A resonance theory of cochlear mechanics. PhD thesis, Australian National University, Canberra. http://hdl.handle.net/1885/49307

Bell, A. (2014). The cochlea, surface acoustic waves, and resonance. In E. Jabbari, D. H. Kim, L. P. Lee, A. Ghaemmaghami, \& A. Khademhosseini (Eds.), Handbook of biomimetics and bioinspiration (pp. 719-741). World Scientific.

Bell, A. (2003). Are outer hair cells pressure sensors? Basis of a SAW model of the cochlear amplifier. In A. W. Gummer (Ed.), Biophysics of the cochlea: From molecules to models (pp. 429-431). World Scientific.

Bell, A. (2008). The pipe and the pinwheel: Is pressure an effective stimulus for the 9+0 primary cilium? Cell Biology International, 32, 462-468.

Bell, A. (2011). How do middle ear muscles protect the cochlea? Reconsideration of the intralabyrinthine pressure theory. Journal of Hearing Science, 1(2), 9-23.

Bell, A. (2012). A resonance approach to cochlear mechanics. PLoS ONE, 7, e47918. https://doi.org/10. 1371/journal.pone.0047918

Bell, A., \& Fletcher, N. H. (2004). The cochlear amplifier as a standing wave: "Squirting" waves between rows of outer hair cells? Journal of the Acoustical Society of America, 116, 1016-1024. https://doi.org/ 10.1121/1.1766053

Benedetto, J. J., \& Teolis, A. (1993). A wavelet auditory model and data compression. Applied and Computational Harmonic Analysis, 1(1), 3-28.

Bialek, W. S. (1983a). Quantum noise and active feedback. Physical Review D, 28, 2096-2098.

Bialek, W. S. (1983b). Thermal and quantum noise in the inner ear. In E. de Boer \& M. A. Viergever (Eds.), Mechanics of hearing (pp. 185-192). Delft University Press.

Bialek, W. S. (1983c). Thermal noise and active processes in the inner ear: Relating theory to experiment. In R. Klinke \& R. Hartmann (Eds.), Hearing: Physiological bases and psychophysics (pp. 51-57). Springer.

Bialek, W. S. (1984). Phonon super-radiance. Physics Letters, 103A, 349-352.

Bialek, W. S. (1987). Physical limits to sensation and perception. Annual Review of Biophysics and Biophysical Chemistry, 16, 455-478.

Bialek, W. (2012). Biophysics: Searching for principles. Princeton University Press.

Bialek, W. S., \& Schweitzer, A. (1985). Quantum noise and the threshold of hearing. Physical Review Letters, 54, 725-728.

Bialek, W. S., \& Wit, H. P. (1984). Quantum limits to oscillator stability: Theory and experiments on acoustic emissions from the human ear. Physics Letters, 104A, 173-178. https://doi.org/10.1016/03759601(84)90371-2

Bruna, J. (2012). Scattering representations for recognition. PhD thesis, Ecole Polytechnique, Paris.

Chalmers, D. J. (1996). The conscious mind: In search of a fundamental theory. Oxford University Press.

Cooper, N. P., \& Guinan, J. J. (2011). Efferent insights into cochlear mechanics. In C. A. Shera \& E. S. Olson (Eds.), What fire is in mine ears: Progress in auditory biomechanics (pp. 396-402). American Institute of Physics. 
Costa, A. R., Pinto-Costa, R., Sousa, S. C., \& Sousa, M. M. (2018). The regulation of axon diameter: From axonal circumferential contractility to activity-dependent swelling. Frontiers in Molecular Neuroscience, 11, 319. https://doi.org/10.3389/frmol.2018.00319

Dallos, P. (1996). Overview: Cochlear neurobiology. In P. Dallos, A. N. Popper, \& R. R. Fay (Eds.), The cochlea (pp. 1-43). Springer.

Dallos, P. (2003). Organ of Corti kinematics. Journal of the Association for Research in Otolaryngology, 4, 416-421.

Dornhoffer, J., Danner, C., Mennemeier, M., Blake, D., \& Garcia-Rill, E. (2006). Arousal and attention deficits in patients with tinnitus. International Tinnitus Journal, 12, 9-16.

Eccles, J. (1990). A unitary hypothesis of mind-brain interaction in the cerebral cortex. Proceedings of the Royal Society of London B, 240, 433-451.

Eccles, J. C. (1994). How the self controls its brain. Springer-Verlag.

Ehm, W. (2010). Broad views of the philosophy of nature: Riemann, Herbart, and the "matter of the mind." Philosophical Psychology, 23, 141-162.

Fechner, G. T. (1860). Elements of psychophysics (trans. H. E. Adler). Holt, New York.

Gallagher, R. (1984). Riemann and the Göttingen school of physiology. Fusion, 6(3), 24-30.

Gellé, M. (1881). Les lésions nerveuses dans la surdité Transactions. International Congress of Medicine, 3, 370-372.

Georgiev, D. D. (2018). Quantum information and consciousness: A gentle introduction. CRC Press.

Gold, T. (1989). New ideas in science. Journal of Scientific Exploration, 3, 103-112.

Goldstein, J. L. (1967). Auditory nonlinearity. The Journal of the Acoustical Society of America, 41, $676-689$.

Hartmann, W. M. (1998). Signals, sound, and sensation. Springer.

Heidelberger, M. (2004). Nature from within: Gustav Theodor Fechner and his psychophysical worldview (trans. Cynthia Klohr). Pittsburgh: University of Pittsburgh Press.

Helmholtz, H. L. F. (1875). On the sensations of tone as a physiological basis for the theory of music. London: Longmans, Green.

Helmholtz, H. L. F. (1911). Handbuch der physiologischen Optik. Dritte Auflage ergänzt und herausgegeben in Gemeinschaft mit A. Gullstrand und J. von Kriess von W. Nagel. Zweiter Band Leopold Voss, Hamburg und Leipzig (translated as Helmholtz's Treatise on Physiological Optics, Vol. II, ed. J. P. C. Southall, Dover, New York, 1962).

Hergenhahn, B. R. (2009). An introduction to the history of psychology (6th ed.). Wadsworth.

Hudspeth, A. J. (2014). Integrating the active process of hair cells with cochlear function. Nature Reviews Neuroscience, 15, 600-614. https://doi.org/10.1038/nrn3786

Kemp, D. T. (1978). Stimulated acoustic emissions from within the human auditory system. Journal of the Acoustical Society of America, 64, 1386-1391. https://doi.org/10.1121/1.382104

Kemp, D. T. (2008). Otoacoustic emissions: Concepts and origins. In G. A. Manley, R. R. Fay, \& A. N. Popper (Eds.), Active processes and otoacoustic emissions (pp. 1-38). Springer.

Kistler, W. M., \& De Zeeuw, C. I. (2003). Time windows and reverberating loops: A reverse-engineering approach to cerebellar function. The Cerebellum, 2, 44-54.

Lozano-Soldevilla, D., \& VanRullen, R. (2019). The hidden spatial dimension of alpha: 10-Hz perceptual echoes propagate as periodic traveling waves in the human brain. Cell Reports, 26, 374-380. https://doi.org/10.1016/j.celrep.2018.12.058

Mallat, S. (2012). Group invariant scattering. Communications on Pure and Applied Mathematic, 65(10), 1331-1398.

Manley, G. A., \& Brownell, W. E. (2008). Future directions in the study of active processes and otoacoustic emissions. In G. A. Manley, R. R. Fay, \& A. N. Popper (Eds.), Active processes and otoacoustic emissions in hearing (pp. 461-471). Springer.

Manley, G. A., Gummer, A. W., Popper, A. N., \& Fay, R. R. (2017). Understanding the cochlea. Springer.

Neely, S. T. (1983). The cochlear amplifier. In E. De Boer \& M. A. Viergever (Eds.), Mechanics of hearing (pp. 111-118). Delft University Press.

Olson, E. S., Duifhuis, H., \& Steele, C. R. (2012). Von Bekesy and cochlear mechanics. Hearing Research, 293, 31-43.

Pang, X. D., \& Peake, W. T. (1986). How do contractions of the stapedius muscle alter the acoustic properties of the ear? In J. B. Allen, J. L. Hall, A. E. Hubbard, S. T. Neely, \& A. Tubis (Eds.), Peripheral auditory mechanisms (pp. 36-43). Springer.

Papadopoulos, A. (2017). Looking backward: From Euler to Riemann. In L. Ji, A. Papadopoulos, \& S. Yamada (Eds.), From Riemann to differential geometry and relativity (pp. 1-94). Springer. 
Parpart, U. (1979). Riemann declassified: his method and program for the natural sciences. Fusion, 2(6), 24-37.

Pesic, P. (2013). Helmholtz, Riemann, and the sirens: Sound, color, and the "problem of space." Physics in Perspective, 15, 256-294.

Pesic, P. (2014). Riemann and the sound of space. Music and the making of modern science (pp. 231307). MIT Press.

Plotnitsky, A. (2017). Comprehending the connection of things: Bernhard Riemann and the architecture of mathematical concepts. In L. Ji, A. Papadopoulos, \& S. Yamada (Eds.), From Riemann to differential geometry and relativity (pp. 329-363). Springer.

Popper, K., \& Eccles, J. C. (1977). The self and its brain: An argument for interactionism. Springer.

Raufer, S., Guinan, J. J., \& Nakajima, A. (2019). Cochlear partition anatomy and motion in humans differ from the classic view of mammals. Proceedings of the National Academy of Sciences, 116, 1397713982. https://doi.org/10.1073/pnas.1900787116

Riemann, B. (1900). On psychology and metaphysics: being the philosophical fragments of Bernhard Riemann (translation by C. J. Keyser from Riemann's Gesammelte Mathematische Werke). The Monist, 10, 198-215. https://doi.org/10.5840/monist19001029

Riemann, B. (1984). The mechanism of the ear (translation of Mechanik des Ohres, 1866, by David Cherry, Robert Gallagher, and John Sigerson). Fusion, 6(3), 31-38.

Riemann, B. (1990). Gesammelte mathematische werke. In H. Weber (Ed.), Collected papers. Berlin: Springer.

Riemann, B. (1995). Philosophical fragments (translation by D. Cherry from Riemann's Gesammelte mathematische Werke). 21st Century Science \& Technology, 8(4), 50-62.

Ritchey, T. (1991). On scientific method: Based on a study by Bernhard Riemann. Systems Research, 8(4), 21-41.

Rosenblum, B., Kuttner, F., \& Stapp, H. (2017). Quantum physics of consciousness. Cosmology Science Publishers.

Scholz, E. (1982). Herbart's influence on Bernhard Riemann. Historia Mathematica, 9, 413-440.

Slepecky, N. B. (1996). Structure of the mammalian cochlea. In P. Dallos, A. N. Popper, \& R. R. Fay (Eds.), The cochlea (pp. 44-129). Springer.

Szentágothai, J. (1979). Local neuron network of the neocortex. In F. O. Schmitt \& F. G. Worden (Eds.), The neurosciences fourth study program (pp. 399-415). MIT Press.

Szentágothai, J. (1984). Downward causation? Annual Review of Neuroscience, 7, 1-11.

Wever, E. G. (1949). Theory of hearing. Dover.

Yang, X., Wang, K., \& Shamma, S. A. (1992). Auditory representations of acoustic signals. IEEE Transactions on Information Theory, 38(2), 824-839.

Publisher's Note Springer Nature remains neutral with regard to jurisdictional claims in published maps and institutional affiliations.

Andrew Bell is a biophysicist with interests in hearing, philosophy of science, music, and the phenomena of life, broadly interpreted. He has a BSc in physics and a BA in philosophy of science from the University of Melbourne, and an MSc and PhD in hearing science from The Australian National University. He has worked for CSIRO, Australia's national scientific research organisation, first in experimental roles and then as a science writer. He is now an honorary senior lecturer at the Eccles Institute of Neuroscience in the John Curtin School of Medical Research, where he focuses on theoretical issues in hearing. He is consulting editor for the Journal of Hearing Science. He lives and works in Canberra where he receives inspiration from the beauty of Ngunnawal country.

Bryn Davies is an applied mathematician who studies wave propagation in complex media, with a particular interest in biological and bio-inspired systems. He has an MMath in mathematics from the University of Oxford and a Doctor of Science in mathematics from ETH Zurich. His work uses boundary integral methods and asymptotic techniques to study the behaviour of systems of subwavelength resonators. He has used this theory to study designs of metamaterials with applications in sensing and waveguiding. This includes designing devices that mimic the function of the cochlea. 
Habib Ammari is a Professor of Applied Mathematics at ETH Zürich. Before moving to ETH, he was a Director of Research at the Department of Mathematics and Applications at Ecole Normale Supérieure in Paris. He received a Bachelor's degree in 1992, a Master's degree in 1993, and a PhD in applied mathematics in 1995, all from the Ecole Polytechnique, France. Following this, he received a Habilitation degree in Mathematics from the University of Pierre \& Marie Curie in Paris three years later. Habib Ammari is a world-leading expert in wave propagation phenomena in complex media, mathematical modelling in photonics and phononics, and mathematical biomedical imaging. He has published more than two hundred research papers, eight high profile research-oriented books and edited eight books on contemporary issues in applied mathematics. He was named the 2013 winner of the Kuwait Prize in Basic Sciences and was the recipient of the Khwarizmi International Award in Basic Sciences in 2015. Habib Ammari has been a fellow of the Tunisian Academy of Sciences, Letters and Arts since 2015, of the European Academy of Sciences since 2018, and the Academia Europaea since 2021. 